\title{
Prevalence of HIV and Associated Factors among Infants Born to HIV Positive Women in Amhara Region, Ethiopia
}

\author{
Zelalem Berhan1, Fantu Abebe ${ }^{2 *}$, Molla Gedefaw ${ }^{3}$, Mulugeta Tesfa ${ }^{4}$ \\ ${ }^{1}$ Ethiopian Network of HIV/AIDS Treatment-Care and Support Project, Management Science for Health (MSH), \\ Amhara Regional Office, Bahir Dar, Ethiopia \\ ${ }^{2}$ Human Resource for Health, Amhara Regional Health Bureau, Bahir Dar, Ethiopia \\ ${ }^{3}$ GAMBY College of Medical Science, Bahir Dar, Ethiopia \\ ${ }^{4}$ Department of Public Health, Debremarkos University, Debremarkos, Ethiopia \\ Email: zelalemberhan@yahoo.com, ${ }^{*}$ fantuabebe@gmail.com, mollagedefaw@yahoo.com, \\ mulugetatesfa27@yahoo.com
}

Received 5 March 2014; revised 2 April 2014; accepted 20 April 2014

Copyright ( 2014 by authors and Scientific Research Publishing Inc.

This work is licensed under the Creative Commons Attribution International License (CC BY).

http://creativecommons.org/licenses/by/4.0/

(c) (i) Open Access

\section{Abstract}

Background: An estimated 1.2 percent of pregnant women are living with HIV in Ethiopia and sadly, one of every 3 children born to these women is infected with HIV. Elimination of these mother-to-child transmissions (MTCT) of HIV is possible through HIV testing during pregnancy and taking antiretroviral medications. However, only 24 percent of pregnant women living with HIV have yet received the medication needed to prevent MTCT of HIV in Amhara region, Ethiopia. Hence, there exists a concern that the rate of HIV infection among infants born to HIV positive mothers might be high. This study assessed the prevalence of HIV infection and associated factors among infants born to women living with HIV. Methods: All eligible records of HIV-exposed infants enrolled between January and December 2012 were reviewed from 17 health facilities which were providing PMTCT services. The study included 434 HIV-exposed infants having HIV DNA/PCR test result. Data were collected using structured data extraction formats. Data were then entered into EPI INFO Version 3.5.1 and analyzed by SPSS Version 16.0. Both bivariate and multivariate analyses were carried out to calculate odds ratios and to control for the effect of confounding. Result: The prevalence of HIV among exposed infants was $10.1 \%$. Delay in HIV diagnosis (AOR = 1.3), mixed infant feeding $(A O R=8.8)$, failure to receive antiretroviral therapy during pregnancy or breast feeding $(A O R=21.6)$, failure to attend skilled delivery service $(A O R=6.1)$ and shorter duration of HIV treatment $(A O R=12)$ were the factors that increase the risk of mother-to-child transmission of HIV in this study. Conclusion: The prevalence of HIV among infants born to HIV positive women in the region is close to the national estimate of $10.9 \%$. Delayed HIV diagnosis, 
inadequate use of antiretroviral therapy and lack of skilled delivery care were the factors that enhance mother-to-child transmission of HIV. Having such a significant figure in the era of the world's pledge to eliminate MTCT of HIV is unbearable. Ensuring access and use of integrated PMTCT services should be the top priorities of the national PMTCT program.

\section{Keywords}

\section{Antiretroviral Therapy, HIV-Exposed Infants, DNA/PCR, PMTCT, Prevalence of HIV}

\section{Introduction}

Globally, about 330,000 children were infected with HIV in 2011, with over 90\% of these infections occurring in sub-Sahara Africa, and mainly through mother-to-child transmission [1]. Twenty-two countries account for more than $90 \%$ of the global burden, and Ethiopia is one of these priority countries where one of every 3 children born to women living with HIV still gets infected with HIV [1]-[3].

Disease progression is aggressive in the first months of life in infants who acquired the infection in utero or around the time of delivery [4]. If left untreated, almost half of these children will die before turning two years of age and $75 \%$ by the age of five years. Most of these deaths in children with HIV could have been avoided through early infant diagnosis (EID) and provision of effective care and treatment. Interventions like the use of Antiretroviral (ART) drugs by infected pregnant women, safe delivery practices and safe infant feeding have helped reduce the risk of transmission to infants (from $40 \%$ to 5\%) [1] [5].

Cognizant of this, the government of Ethiopia has been accelerating to expand the Prevention of Mother-To-Child Transmission (PMTCT) of HIV service by endorsing with antenatal care free of charge. To eliminate infection in children and keep mothers alive, a comprehensive package of interventions, including, but not limited to, preventing women from becoming infected with HIV, eliminating Mother-To-Child Transmission (MTCT) throughout pregnancy, delivery and breastfeeding by providing effective medications and providing appropriate HIV treatment, care and support for mother and children have been underscored [6].

Moreover, since March 2013, all pregnant women have been considered eligible to start long-term antiretroviral therapy (ART), through a package known as option $\mathrm{B}^{+}$, which has a great role to ensure prevention of at least $98 \%$ of mother-to-child transmission of HIV [5] [6].

Despite the availability and scale-up of these life-saving interventions, only $24 \%$ of 13,000 pregnant women living with HIV in Ethiopia have been receiving the medication to prevent mother-to-child transmission of HIV [1] [3]. Even those who utilize skilled delivery services (12\%) [2], most often go untested for HIV, with devastating consequences for themselves and their newborns. As a result of this, there is a growing concern that the risk of mother-to-child transmission of HIV in the region, perhaps in the zone may be increasing [1] [3].

Early diagnosis of infants infected with HIV followed by prompt ART treatment can help reduce morbidity and mortality. Failing to do that, disease progression and death are usually rapid: almost $50 \%$ of the children infected during pregnancy or delivery die within one year, and about $50 \%$ of children infected during breastfeeding die within nine years of infection [6] [7].

In infants, antibody testing to diagnose HIV infection is inadequate as persistent passively acquired maternal antibodies in the infant may yield false-positive results for up to 18 months or longer. It is, therefore, important to provide accurate diagnostic services for identification of infants infected with HIV. Because of its high sensitivity and specificity, DNA polymerase chain reaction (PCR) has been widely used for diagnosis of HIV amongst exposed infants. The technology allows for PCR being performed using a small spot from a Dried Blood Spot (DBS) sample, as well as identification of infection from birth [7]. As this molecular test has been successfully established in major regions Ethiopia, we only enrolled those HIV-exposed and DBS-tested infants in the zone.

In Ethiopia, few studies [7] [8] tried to demonstrate the risk of mother-to-child transmission of HIV. However, they were either confined to a single facility or assessed the risk among urban dwellers only or using relatively remote data. Assessing the risk and determinant factors in an entire zonal administration that involve both urban and rural families, enrolling all health facilities providing PMTCT service and using relatively latest data availa- 
ble would however justify the essence of this research. In this study, we aimed to determine the prevalence and associated factors of HIV infection among HIV-exposed infants in South Gondar zone, Amhara Ethiopia.

\section{Methods}

\subsection{Study Design}

Facility based cross-sectional study design was conducted using data from the registration books and follow up log books in the PMTCT and HIV exposed infants' (HEI) follow-up units.

\subsection{Study Settings}

This study was conducted in South Gondar zone of the Amhara national regional state, Northwest Ethiopia. The Amhara region is the second largest and populous regional states in Ethiopia, comprising a total of over 19 million inhabitants. The region is one of the hardest hit regions by the burden HIV/AIDS. The prevalence of HIV ${ }^{1}$ among adults and pregnant women in the region is 1.5 and 0.8 percent respectively [2]. The south Gondar administrative zone is one of the ten Zones in Amhara Region. The zone is inhabitant for about 2.4 million people and is administratively divided into 12 districts and 5 town administrations [9]. The burden of HIV in the zone, if not worse, is not different from the regional estimate.

This zone has one general hospital and 16 health centers, all of which provide PMTCT services. The study was conducted in all of these facilities. The data collection was conducted from May to June 2013. The estimated number of pregnant women over the year in the zone was 108,000. However, only $34 \%$ of these women were reportedly attended Antenatal Care Visit (ANC) over the given year (2012). Among a total of 36,720 pregnant women who had at least one ANC during 2012, 455 women were HIV positive, giving rise to a prevalence of HIV among these pregnant women $1.2 \%$.

\subsection{Study Populations}

The study populations were HIV-exposed infants born from those HIV positive pregnant women and enrolled in the health facilities providing PMTCT services in the zone. The data were extracted from all registered HIV exposed and DNA/PCR tested infant charts documented from January $1^{\text {st }}$ to $31^{\text {st }}$ December 2012.

Records were considered eligible for inclusion if the infant's age at diagnosis was recorded, if both infant's and maternal HIV treatment or prophylaxis data are available, and if information is available on infant feeding practice, place of delivery and DNA/PCR test result.

As the number of charts representing the enrolled children for HIV care and support services in the zone was manageable, we included all of the charts. Therefore, the total number of charts reviewed was 455 . However, only 434 charts were having the desired information and included for further analysis.

Because of its high sensitivity and specificity, DNA polymerase chain reaction (PCR) has been widely used for diagnosis of HIV amongst exposed infants. The technology allows for PCR to be performed using a small spot from a dried blood spot (DBS) sample, as well as identification of infection from birth.

Ethiopia has a tiered laboratory network, which is a hierarchical or ladder-like system with the national reference laboratory at the top, followed by regional, referral and/or specialized hospital laboratories. Once DBS are collected it will be transported and tested in the regional laboratories and the results will be returned to the facilities where the samples were collected.

Being the Gold standard test to determine HIV infection, DNA PCR testing facilities have been expanded even in the region. Following the expansion of HIV DNA PCR testing facilities in the region, DBS samples haves have been collected and tested for all infants born to HIV positive pregnant women. Since the test is strong enough to determine the infection rate with a single test, we only used the results of PCR for HIV DNA antigen in DBS samples of HEIs.

DNA/PCR test result was the dependent variable, while characteristics that might determine the risk of HIV infection such as time of HIV testing, intake of prophylaxis by infants, maternal ART or ARV prophylaxis intake, place of delivery, infant feeding practice, maternal education, monthly income and duration of treatment were the independent variables.

A structured data extraction tool was developed, pre-tested and used to collect the information from the ${ }^{1}$ Prevalence of HIV among HIV exposed infants and associate factors. 
charts. The tool was adapted from the national standard HIV exposed infant follow up chart and PMTCT registration log book, which comprises, socio-demographic characteristics, information on ARV prophylaxis for the mother and infant, place of delivery and infant feeding practice and some other important variables. One ART trained nurse and one ART trained Health officer or midwife in each health facility were recruited to collect the data.

One day training was given for data collectors and supervisors. The collected data were reviewed daily for completeness, accuracy, clarity, and consistency by the supervisors and the principal investigators. Then codes were given to the completed questionnaires. The data were cleaned and edited before entered to EPI-info Version 3.5.2. After the data were checked for correct entry we exported it to SPSS version 16 software for analysis. Further data cleaning and frequency run was made to check for accuracy, outliers, consistencies and missed values and variables. Any error identified was corrected.

Frequency run, cross tabulations and summary statistics were used to describe the study population in relation to relevant variables. Crude and adjusted odds ratios were computed to assess presence of association and the magnitude of statistical significance. $P$ value $\leq 0.05$ at $95 \%$ CI was considered statistically significant. Binary logistic regression analysis was employed to see the effect of each independent variable on the outcome variable and to control for the effect of confounding.

\subsection{Ethical Consideration}

Ethical clearance was obtained from Debre Markos University ethical clearance committee. Official letter was also sought from Amhara regional state health bureau and South Gondar zonal department. The zonal health department and each district health office offered letters to inform in charges of all eligible health facilities. Since we used secondary data, we did not require informed consent from the clients. The data retrieved from each health center's HIV exposed infant records and PMTCT registration log books were entirely used anonymously as it does not bear any name or identification number. The collected data are kept strictly confidential.

\section{Result}

Among the total of 455 reviewed HIV exposed infants' (HEI) and HIV positive pregnant women records, 434 charts were found to have complete information hence were considered for the study.

\subsection{Socio-Demographic Characteristics of the Study Participants}

Female HEI infants constitute 51.6\%, whereas the remaining $48.4 \%$ were males. Age at the time of enrollment for HIV care and support for the majority (60.6\%) of children was at six weeks.

\subsection{Findings on HIV Care and Support}

Of the total participants, nearly $93 \%$ of the mothers were enrolled in HIV care and support service during the last pregnancy. Among these enrolled $402 \mathrm{HIV}$ positive pregnant women, most of them (63.4\%) were taking highly active ART and 30.3\% were put on ARV prophylaxis. Three hundred twenty eight (75.6\%) infants were born in health facilities. Moreover, 351 (81\%) of these newborns were given ARV prophylaxis right after birth. Pertaining to the duration of ART treatment a pregnant woman had, 338 (89.7\%) mothers had received the treatment for longer than four weeks (Table 1). Among infants who received ARV prophylaxis, the majority (40.2\%) had taken single dose Neverapin and AZT for seven days followed by 30.2\% that had daily NVP for 45 days.

Pertaining to breast feeding, 402 (92.6\%) infants were given exclusive breast feeding, whereas 32 (7.4\%) were put on a mixed feeding. At the time of the study, 390 (89.9\%) infants were on active follow up, whereas 32 (7.4\%) of children were lost to follow up and 12 (2.8\%) children were dead.

With regard to the time of diagnosis of infants born from HIV positive mothers, the majority 324 (74.7\%) had their DBS tested after 6 weeks. While only 110 (25.3\%) infants were tested at the right time, which is at 6 weeks (Table 2). The mean age at which DBS test done was 17 weeks.

The prevalence of DNA/PCR positivity among children born to HIV positive mothers was $10.1 \%$ (44 out of 434 infants tested positive) (Figure 1), with a 95\% confidence interval between $7.3 \%$ and $13 \%$. The risk of HIV 
Table 1. Socio-demographic characteristics of HIV exposed infants and their mothers, South Gondar, Amhara, Ethiopia $(\mathrm{n}=$ 434), July 2013.

\begin{tabular}{|c|c|c|}
\hline Variables & Frequency & Percentage \\
\hline \multicolumn{3}{|l|}{ Sex of the infant } \\
\hline Male & 210 & 48.4 \\
\hline Female & 224 & 51.6 \\
\hline \multicolumn{3}{|l|}{ Age of the mother } \\
\hline $15-24$ & 95 & 21.9 \\
\hline $25-34$ & 260 & 69.9 \\
\hline$\geq 35$ & 79 & 18.4 \\
\hline \multicolumn{3}{|l|}{ Marital status of the mother } \\
\hline Currently married & 373 & 85.9 \\
\hline Currently not married & 61 & 14.1 \\
\hline \multicolumn{3}{|c|}{ Educational status of the mother } \\
\hline Unable to read and write & 251 & 57.8 \\
\hline Able to read and write & 183 & 42.2 \\
\hline \multicolumn{3}{|l|}{ Occupational status of mother } \\
\hline Housewife & 290 & 66.8 \\
\hline Governmental employed & 22 & 5.1 \\
\hline Unemployed & 122 & 28.1 \\
\hline \multicolumn{3}{|c|}{ Number of children a mother has } \\
\hline $1-3$ & 369 & 85 \\
\hline 4 and above & 65 & 15 \\
\hline
\end{tabular}

Table 2. Findings on HIV care and support of HIV exposed infants and their mothers, South Gondar, Amhara, Ethiopia (n = 434), July 2013.

\begin{tabular}{|c|c|c|}
\hline Variables & Frequency & Percentage \\
\hline \multicolumn{3}{|l|}{ Infant's age at diagnosis } \\
\hline At 6 weeks & 110 & 25.3 \\
\hline After 6 weeks & 324 & 74.7 \\
\hline \multicolumn{3}{|c|}{ Infants received CPT at 6 weeks of birth } \\
\hline Yes & 377 & 86.9 \\
\hline No & 57 & 13.1 \\
\hline \multicolumn{3}{|c|}{ Infants received ARV prophylaxis at birth } \\
\hline Yes & 351 & 80.9 \\
\hline No & 83 & 19.1 \\
\hline \multicolumn{3}{|l|}{ Infants feeding practice } \\
\hline Exclusive Breast feeding & 402 & 92.6 \\
\hline Replacement feeding & 0 & 0 \\
\hline Mixed feeding & 32 & 7.4 \\
\hline \multicolumn{3}{|c|}{ Mothers enrolled in HIV/ART care } \\
\hline Yes & 402 & 92.6 \\
\hline No & 32 & 7.4 \\
\hline \multicolumn{3}{|c|}{ Mother received ART/ARV prophylaxis $(n=402)$} \\
\hline ART & 255 & 63.4 \\
\hline ARV & 122 & 30.3 \\
\hline None & 25 & 6.2 \\
\hline
\end{tabular}




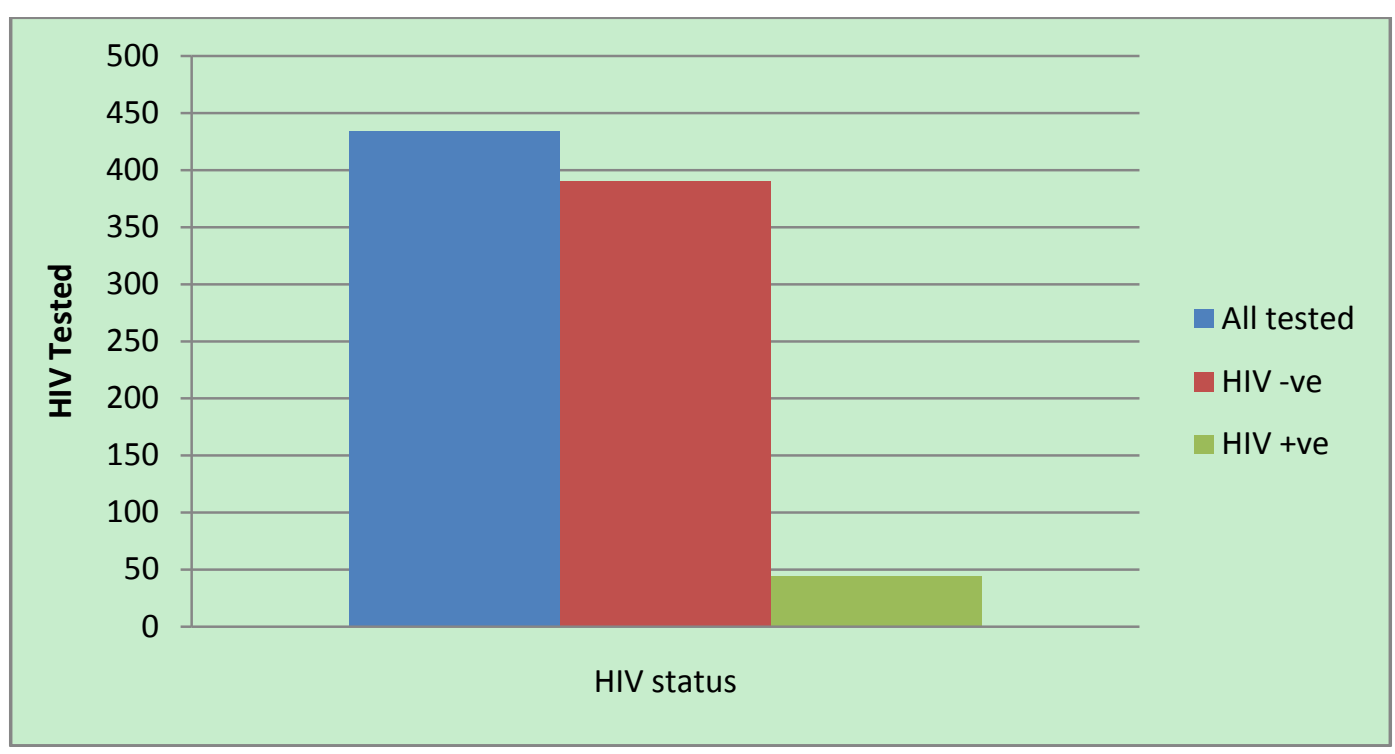

Figure 1. Result of HIV testing among HIV exposed infants July 2013, Amhara, Ethiopia.

infection between males and female infants was roughly equal, $10.5 \%$ and $9.8 \%$ respectively.

\subsection{Bivariate Analysis of Factors Associated with DNA/PCR Result}

Infants whose DBS tested after 6 weeks were more likely to have positive HIV result than otherwise (OR $=2.3$, $95 \% \mathrm{CI}=1.1,4.9)$. Infants born from a mother with an age group between 25 - 34 years were more likely to be positive $(\mathrm{OR}=1.4,95 \% \mathrm{CI}=0.6,4.9)$ than the other age categories; however, it was not statistically significant.

Infants born from non-educated mothers tend to be positive than those having educated mothers $(\mathrm{OR}=8.4$, $95 \% \mathrm{CI}=2.9,14.8)$. All DNA/PCR positive infants were born from mothers having few $(1-3)$ numbers of children, but it has no statistically significant association.

DNA/PCR positivity was higher among infants who did not receive ARV prophylaxis or ART at all (COR = 10.6, 95\% CI $=3.5,13.8$ ), but no difference was observed between different categories prophylaxis either ARV or ART and HIV test result.

Those infants having a mixed feeding in the first six months of their life were found to be more DNA/PCR positive than those received exclusive breastfeeding $(\mathrm{COR}=12.4,95 \% \mathrm{CI}=6.0,18.1)$. Mothers who were not enrolled to HIV care and support service had relatively higher risks of giving birth of DNA/PCR positive infants than their counterparts $(\mathrm{COR}=6.9,95 \% \mathrm{CI}=3.1,15.4)$.

Infants born from mothers who received neither ART nor ARV prophylaxis were more likely to be DNA/PCR positive than otherwise (COR $=16.0,95 \% \mathrm{CI}=6.8,24.9)$. Moreover, infants were more likely to be DNA/PCR positive if their mothers took the ART or ARV prophylaxis for less than 4 weeks $(\mathrm{COR}=23.8,95 \% \mathrm{CI}=8.8$, 39.0). More infants born at home were more likely to be DNA/PCR positive than those born in health facility $(\mathrm{COR}=8.8,95 \% \mathrm{CI}=4.4,17.5)$ (Table 3$)$.

\subsection{Multivariate Analysis of Factors Associated with DNA/PCR Result}

We entered variables having $P$ value $<0.2$ in the bivariate analysis to the final model.

Age at DBS tested was significantly associated with HIV test results, those having DBS test after 6 weeks were more likely to have positive DNA/PCR result $(\mathrm{AOR}=1.3,95 \% \mathrm{CI}=1.1,10.1)$ than their counterparts.

Receiving ARV prophylaxis was found to be protective of mother to child transmission of HIV in this study, infants who did not receive any prophylaxis were 6.7 times more likely to become HIV positive than protected children (AOR $=6.7,95 \% \mathrm{CI}=2.9,23.1$ ). Mixed feeding was significantly associated with positive HIV test result ( $\mathrm{AOR}=8.8,95 \% \mathrm{CI}=4.5,22.8$ ). Mother to child transmission was higher if the mother did not receive either ART or ARV prophylaxis during pregnancy or breastfeeding. If a mother did not receive ART or ARV 
Table 3. Bivariate analysis of factors associated with HIV positivity among infants born to HIV mothers, South Gondar, Amhara, Ethiopia July 2013.

\begin{tabular}{|c|c|c|c|c|}
\hline & \multicolumn{2}{|c|}{ DBS result } & \multirow{2}{*}{ COR $(95 \%$ CI) } & \multirow{2}{*}{$P$ value } \\
\hline & Negative & Positive & & \\
\hline \multicolumn{5}{|l|}{ Age at DBS tested } \\
\hline At 6 months & 104 & 6 & 1 & \multirow{2}{*}{0.002} \\
\hline After 6 months & 286 & 38 & $2.3(1.1,4.9)^{*}$ & \\
\hline \multicolumn{5}{|l|}{ Mothers' age } \\
\hline $15-24$ & 83 & 12 & 1 & \multirow{3}{*}{0.761} \\
\hline $25-34$ & 234 & 26 & $1.7(0.6,4.6)$ & \\
\hline $35+$ & 73 & 6 & $1.3(0.5,3.4)$ & \\
\hline \multicolumn{5}{|l|}{ Maternal education } \\
\hline Unable read \& write & 211 & 40 & $8.4(2.9,14.8)^{*}$ & \multirow{2}{*}{0.013} \\
\hline Able to read \& write & 179 & 4 & 1 & \\
\hline \multicolumn{5}{|c|}{ Infant ARV prophylaxis } \\
\hline Yes & 337 & 14 & 1 & \multirow{2}{*}{0.0001} \\
\hline No & 53 & 30 & $10.6(3.5,13.8)^{*}$ & \\
\hline \multicolumn{5}{|l|}{ Place of delivery } \\
\hline Health facility & 314 & 14 & 1 & \multirow{2}{*}{0.0001} \\
\hline Home & 76 & 30 & $8.8(4.4,17.5)^{*}$ & \\
\hline \multicolumn{5}{|l|}{ Feeding practice } \\
\hline EBF & 374 & 28 & 1 & \multirow{2}{*}{0.0001} \\
\hline MF & 16 & 16 & $12.4(6.0,18.1)^{*}$ & \\
\hline \multicolumn{5}{|l|}{ Enrollment } \\
\hline Yes & 370 & 32 & 1 & \multirow{2}{*}{0.004} \\
\hline No & 20 & 12 & $6.9(3.1,15.4)^{*}$ & \\
\hline \multicolumn{5}{|l|}{ Mothers' treatment } \\
\hline ART & 245 & 10 & 1 & \multirow{3}{*}{0.0001} \\
\hline ARV prophylaxis & 110 & 12 & $2.6(1.1,6.3)^{*}$ & \\
\hline None & 15 & 10 & $16.0(6.8,24.9)^{*}$ & \\
\hline \multicolumn{5}{|l|}{ Duration of treatment } \\
\hline$>4$ weeks & 330 & 8 & 1 & \multirow{2}{*}{0.0001} \\
\hline$\leq 4$ weeks & 25 & 14 & $23(8.8,39)^{*}$ & \\
\hline
\end{tabular}

*Statistically significant at $95 \%$ CI, $P$ value less than 0.05.

prophylaxis at all or if she received for less than 4 weeks, the likelihood that her infant will be HIV positive increased by $21.6 \%$ and $12 \%$ respectively, $(\mathrm{AOR}=21.6,95 \% \mathrm{CI}=14.5,39.8)$ and $(\mathrm{AOR}=12,95 \% \mathrm{CI}=4.2,45)$ (Table 4). Place of delivery was also found to be predictive of mother-to-child transmission of HIV. Those infants born at home were 6 times more affected than infants born at health facility $(\mathrm{AOR}=6.1,95 \% \mathrm{CI}=2.1$, 18.6). 
Table 4. Multivariate analysis of factors associated with HIV positivity among infants born to HIV positive mothers, south Gondar Amhara, July 2013.

\begin{tabular}{|c|c|c|c|c|}
\hline \multirow{2}{*}{ Variables } & \multicolumn{2}{|c|}{ DBS result } & \multirow{2}{*}{ COR (95\% CI) } & \multirow{2}{*}{ AOR (95\% CI) } \\
\hline & Negative & Positive & & \\
\hline \multicolumn{5}{|l|}{ Age at DBS tested } \\
\hline At 6 months & 104 & 6 & 1 & 1 \\
\hline After 6 months & 286 & 38 & $2.3(1.4,4.9)^{*}$ & $1.3(1.1,10.1)^{*}$ \\
\hline \multicolumn{5}{|l|}{ Maternal age } \\
\hline $15-24$ & 83 & 12 & 1 & 1 \\
\hline $25-34$ & 234 & 26 & $1.7(0.6,4.6)$ & $1.3(0.2,6.7)$ \\
\hline $35+$ & 73 & 6 & $1.3(0.5,3.4)$ & $0.5(0.1,3.0)$ \\
\hline \multicolumn{5}{|l|}{ Maternal education } \\
\hline Unable read \& write & 211 & 40 & $8.4(2.9,14.8)^{*}$ & $4.2(0.8,21.4)$ \\
\hline Able to read \& write & 179 & 4 & 1 & 1 \\
\hline \multicolumn{5}{|l|}{ Place of delivery } \\
\hline Health facility & 337 & 14 & 1 & 1 \\
\hline Home & 53 & 30 & $10.6(3.5,13.8)^{*}$ & $6.1(2.1,18.6)^{*}$ \\
\hline \multicolumn{5}{|l|}{ Infant ARV prophylaxis } \\
\hline Yes & 314 & 14 & 1 & 1 \\
\hline No & 76 & 30 & $8.8(4.4,17.5)^{*}$ & $6.7(2.9,23.1)^{*}$ \\
\hline \multicolumn{5}{|l|}{ Feeding practice } \\
\hline $\mathrm{EBF}$ & 374 & 28 & 1 & 1 \\
\hline MF & 16 & 16 & $12.4(6.0,18.1)^{*}$ & $8.8(4.5,22.8)^{*}$ \\
\hline \multicolumn{5}{|l|}{ Enrollment } \\
\hline Yes & 370 & 32 & 1 & 1 \\
\hline No & 20 & 12 & $6.9(3.1,15.4)^{*}$ & $2.5(0.6,10.1)$ \\
\hline \multicolumn{5}{|l|}{ Mothers' treatment } \\
\hline ART & 245 & 10 & 1 & 1 \\
\hline ARV prophylaxis & 110 & 12 & $2.6(1.1,6.3)^{*}$ & $3.1(0.7,12.8)$ \\
\hline None & 15 & 10 & $16.0(6.8,24.9)^{*}$ & $21.6(14.5,39.8)^{*}$ \\
\hline \multicolumn{5}{|l|}{ Duration of treatment } \\
\hline$>4$ weeks & 330 & 8 & 1 & 1 \\
\hline$\leq 4$ weeks & 25 & 14 & $23(8.8,39)^{*}$ & $12(4.2,45)$ \\
\hline
\end{tabular}

*Statistically significant at $95 \%$ CI, $P$ value less than 0.05 : adjusted for age at DBS tested, maternal age, maternal education, feeding practices, place of delivery and prophylaxis. 
However, maternal education, and history of enrollment that had significant association in the bivariate analysis lost their significance when controlled for confounding factors in the final model.

\section{Discussion}

The major aim of this study was to assess the prevalence of HIV infection and its correlates among infants born to HIV infected mothers in South Gondar Zone, Northwest Ethiopia.

In this study, the prevalence of HIV infection among HIV exposed infants was $10.1 \%$, which is almost close to the national estimate of $10.9 \%$ [3]. Having a number of interventions implemented to reduce the prevalence of mother to child transmission of HIV, the result in this study showed higher number. Despite the World's pledge to eliminate MTCT of HIV by December 31st, 2015 [5], the finding indicated that the progress we have made so far to produce an HIV free generation is very slow.

This study demonstrated that a number of factors are related to MTCT of HIV. Consistent with other studies [10]-[13], infants having their DBS test done after 6 weeks of their age were more likely to be HIV positive than infants tested at the age of $6^{\text {th }}$ weeks. This observation may be due to the latter infants appearing for diagnosis; the more likely they would be exposed to HIV infection as transmission through breastfeeding is common. The risk might have coupled if a mother is not enrolled in HIV care and support during pregnancy or post-partum or if mixed feeding would be practiced. Maternal ART and infants' ARV prophylaxis are effective interventions to curb MTCT of HIV during this time [3] [5].

Infants born at home were 6.7 times more likely to be HIV positive than those born at the health facility. This is because of the fact that women attending skilled delivery service would be given antiretroviral treatment or antiretroviral prophylaxis to prevent mother to child transmission of HIV during labor and delivery. Even, HIV exposed newborns would have the opportunity to receive ARV prophylaxis immediately, thereby minimizing the risk of acquiring HIV infection during labor and delivery as the highest proportion of newborns are infected during this time. This finding is consistent with studies done in Ethiopia and Tanzania [13]-[15]. However, in this study an appreciable number of pregnant women did not attend skilled delivery care, leaving the devastating effects of HIV to their children. Seamless and concerted efforts are needed to ensure all pregnant women are receiving skilled delivery services.

Those infants born from mothers who did not receive either ART or ARV prophylaxis were having a greater risk of HIV infection. The fact that taking ART could reduce maternal viral load and subsequent transmission of the virus to the fetuses or newborns; those having no history of treatment were more likely to infect their babies than their counterparts. This finding is consistent with studies elsewhere [13] [15] [16]. Without treatment, up to $40 \%$ of babies born to HIV positive mothers will start life infected, and almost half of these will die before they are two years old [4] [5]. Therefore, there should not be any reluctance or missed opportunity to enroll all HIV positive pregnant women for available HIV care and support service.

Another predictor of mother to child transmission of HIV in this study was infanthood ARV prophylaxis; those infants who did not receive ARV prophylaxis right after birth were at risk of acquiring HIV infection. This might be due to the effect of these drugs to eliminate the virus in the newborn's blood. In fact this was in agreement with studies done in Ethiopia and Zambia [13] [14] but not with others [16] [17].

Consistent with studies done in Ethiopia [13] [15] and Kenya [17], infants who received mixed feeding were 9 times more likely to acquire HIV infection than their counterparts. This would be due to the risk of mixed feeding to result in gastrointestinal infection and laceration that leads to mucosal barrier breakage and subsequent promotion of viral entry to the blood stream and progression of HIV infection. Lack of knowledge of mothers on the adequacy of breast feeding to satisfy nutritional demands of their infants ( $<6$ months) might contribute to the observed feeding malpractice. There might be missed opportunities to advise mothers' of HEIs on infant feeding during antepartum or postpartum period. As breast feeding is the most preferred nutritional option for HEIs younger than 6 months [6], we have to make sure that every mother has the right information and means to make appropriate decisions.

Moreover, duration of ART treatment also matter the probability of infection, the longer ( $>4$ weeks) women were on ART, the lower chance they have to transmit HIV to their babies. This observation may be due to the effect of the treatment on the viral load reduction and subsequent transmission. This finding is in agreement with other studies [15] [16] [18] [19]. This finding underscores the critical need of earlier diagnosis and timely treatment of HIV among pregnant women. This would eventually ensure elimination of MTCT of HIV in the region. 
Unlike other previous studies [8] [13] maternal education and time of enrollment of children for HIV care and support did not show a statistically significant relationship with the outcome variable in the final model. This variation may be due to the difference in sample size, study area or study design.

\section{Limitation of the Study}

As this research was conducted using secondary data, it was impossible to obtain some essential information in the charts, such as household monthly income, maternal viral load during pregnancy and whether she was infected before or during the last pregnancy. The relatively small sample size might also affect the power of the test. Therefore, generalization of the findings may not be possible. Although this study might suffer from its lower precision, it would be valuable evidence to evaluate program effectiveness and provide a foundation for future intervention.

\section{Conclusion}

The proportion of infected infants born to HIV infected mothers is unacceptably high. Delayed diagnosis, failure to seek skilled delivery service, mixed infant feeding practice and failure to provide at all or shorter duration of HIV treatment were the factors that increased the risk of mother-to-child transmission of HIV. There is a lot to be done, giving that we are on the eve of world's pledge to eliminate MTCT of HIV by December $31^{\text {st }} 2015$. Integrated and audience-specific education and promotion of using comprehensive HIV care and support services should be strengthened. Client centered, friendly and culturally sensitive PMTCT service could help reduce this preventable infection of infants - the future of Ethiopia. Research is needed to explore reasons related to woman's failure to use HIV care and support services in the region. Assessing the risk among private providers may also reveal valuable information.

\section{Acknowledgements}

We are grateful in extending our thanks to Amhara National Regional State (ANRS) health bureau, to all public health facilities in South Gondar administrative zone, to all districts in the zone and all data collectors and supervisors. Our special gratitude goes to the MSH Amhara regional office for funding the research project.

\section{Authors' Contributions}

Z B has participated in the design, coordination of the study and data collection, analysis and interpretation. F A has contributed to data analysis, interpretation of statistical data and draft the manuscript. Both M G and M T have contributed in the design and coordination of the study. All authors read and approved the final manuscript.

\section{References}

[1] WHO, UNAIDS (2012) A Progress Report on the Global Plan towards the Elimination of New HIV Infections among Children by 2015 and Keeping Their Mothers Alive. WHO, UNAIDS, Geneva.

[2] Central Statistical Agency (CSA) (2012) Ethiopian Demography and Health Survey, Addis Ababa, ICF International, Calverton. CSA, Addis Ababa.

[3] Federal HAPCO (2012) Country Progress Report on HIV/AIDS Response, Ethiopia. Federal HAPCO, Addis Ababa.

[4] UNAIDS/WHO (2012) A Progress Report on the Global Plan towards the Elimination of New HIV Infections among Children by 2015 and Keeping Their Mothers Alive. UNAIDS/WHO, Geneva.

[5] Business Leadership Council (2012) For a Generation Born HIV Free; End the Transmission of HIV from Mother-ToChildren by December $31^{\text {st }} 2015$.

[6] WHO (2012) Use of Antiretroviral Drugs for Treating Pregnant Women and Preventing HIV Infection in Infants. WHO, Geneva.

[7] Fonjungo, P.N. (2012) Field Expansion of DNA Polymerase Chain Reaction for Early Infant Diagnosis of HIV-1: The Ethiopian Experience. Center for Global Health, Centers for Disease Control and Prevention (CDC), Atlanta.

[8] Digsu, K., et al. (2013) Mother-To-Child Transmission of HIV and Its Predictors among HIV-Exposed Infants at a PMTCT Clinic in Northwest Ethiopia. BMC Public Health, 13, 398. http://dx.doi.org/10.1186/1471-2458-13-398

[9] Central Statistical Agency of Ethiopia (2007) National HIV Statistics. Addis Ababa. 
[10] WHO (2006) Antiretroviral Therapy for HIV Infection in Infants and Children towards Universal Access. WHO, Geneva.

[11] Federal HIV/AIDS Prevention and Control Office, Federal Ministry of Health (2007) Guidelines for Prevention of Mother-To-Child Transmission of HIV in Ethiopia.

[12] UNAIDS (2010) UNAIDS Report on the Global AIDS Epidemic 2010 Geneva: Joint United Nations Program on HIV/AIDS. UNAIDS, Geneva.

[13] Mirkuzie, A., et al. (2010) Promising Outcomes of a National Program for the Prevention of Mother-To-Child HIV Transmission in Addis Ababa: A Retrospective Study. BMC Health Services Research, 10, 267. http://dx.doi.org/10.1186/1472-6963-10-267

[14] Petra Study Team (2002) Efficacy of Three Short-Course Regimens of Zidovudine and Lamivudine in Preventing Early and Late Transmission of HIV-1 from Mother to Child in Tanzania, South Africa, and Uganda (Petra Study). Lancet, 359, 1178-1186. http://dx.doi.org/10.1016/S0140-6736(02)08214-4

[15] Shargie, T., et al. (2011) Prophylactic Treatment Uptake and Compliance with Recommended Follow Up among HIV Exposed Infants: A Retrospective Study in Addis Ababa, Ethiopia. BMC Research Notes, 4, 563.

[16] Tonwe, G.B., et al. (2009) Implementing Family-Focused HIV Care and Treatment: The First 2 Years' Experience of the Mother-To-Child Program in Abidjan, Cote d'Ivoire. Tropical Medicine \& International Health, 14, $204-212$. http://dx.doi.org/10.1111/j.1365-3156.2008.02182.x

[17] Azcoaga, A., et al. (2011) Effectiveness of a PMTCT Program in Rural Western Kenya. AIDS Care, 23, $274-280$. http://dx.doi.org/10.1080/09540121.2010.507750

[18] WHO/UNICEF (2007) Guideline on Global Scale-Up of the Prevention of Mother-To-Child Transmission of HIV towards Universal Access for Women, Infants and Young Children and Eliminating HIV and AIDS among Children. WHO/UNICEF, Geneva.

[19] WHO (2006) Antiretroviral Drugs for Treating Pregnant Women and Preventing HIV Infection in Infants: Towards Universal Access: Recommendations for a Public Health Approach. WHO, Geneva.

\section{Abbreviations}

Adjusted Odds Ratio (AOR), Antiretroviral Therapy (ART), Antiretroviral (ARV), Zidovudine (AZT), Confidence Interval (CI), Crude Odds Ratio (COR), Dried Blood Spot (DBS), Deoxyribonucleic Acid (DNA), Exclusive Breast Feeding (EBF), Early Infant Diagnosis (EID), HIV Exposed Infants (HEI), Mixed Feeding (MF), Mother to Child Transmission of HIV (MTCT), Neverapin (NVP), Polymerase Chain Reaction (PCR), Prevention of Mother to Child Transmission (PMTCT). 